\title{
Building Resilience against COVID-19 Pandemic Using Artificial Intelligence, Machine Learning, and IoT: A Survey of Recent Progress
}

\author{
S. M. Abu Adnan Abir ${ }^{1}$, Shama Naz Islam ${ }^{2}$, Adnan Anwar ${ }^{3}$, Abdun Naser Mahmood ${ }^{4, * D}$ \\ and Aman Maung Than Oo ${ }^{2}$ (D) \\ 1 IGW Operators Forum, Dhaka 1212, Bangladesh; adnanabir.iut@gmail.com \\ 2 School of Engineering, Deakin University, Geelong, VIC 3216, Australia; shama.i@deakin.edu.au (S.N.I.); \\ aman.m@deakin.edu.au (A.M.T.O.) \\ 3 Strategic Centre for Cyber Security Research \& Innovation (CSRI), School of Information Technology, \\ Deakin University, Geelong, VIC 3216, Australia; adnan.anwar@deakin.edu.au \\ 4 School of Engineering and Mathematical Sciences, La Trobe University, Melbourne, VIC 3086, Australia \\ * Correspondence: A.Mahmood@latrobe.edu.au; Tel.: +61-3-9479-2145
}

Received: 6 November 2020; Accepted: 4 December 2020; Published: 6 December 2020

\begin{abstract}
Coronavirus disease 2019 (COVID-19) has significantly impacted the entire world today and stalled off regular human activities in such an unprecedented way that it will have an unforgettable footprint on the history of mankind. Different countries have adopted numerous measures to build resilience against this life-threatening disease. However, the highly contagious nature of this pandemic has challenged the traditional healthcare and treatment practices. Thus, artificial intelligence (AI) and machine learning (ML) open up new mechanisms for effective healthcare during this pandemic. AI and ML can be useful for medicine development, designing efficient diagnosis strategies and producing predictions of the disease spread. These applications are highly dependent on real-time monitoring of the patients and effective coordination of the information, where the Internet of Things (IoT) plays a key role. IoT can also help with applications such as automated drug delivery, responding to patient queries, and tracking the causes of disease spread. This paper represents a comprehensive analysis of the potential $\mathrm{AI}, \mathrm{ML}$, and IoT technologies for defending against the COVID-19 pandemic. The existing and potential applications of AI, ML, and IoT, along with a detailed analysis of the enabling tools and techniques are outlined. A critical discussion on the risks and limitations of the aforementioned technologies are also included.
\end{abstract}

Keywords: COVID-19; coronavirus; machine learning; artificial intelligence; Internet of Things

\section{Introduction}

Humanity is now encountering a global crisis, perhaps the greatest crisis of this generation: The COVID-19 pandemic. People all over the world came to know this disease first on 31 December 2019, when the Wuhan Municipal Health Commission, China, reported a cluster of cases of pneumonia in Wuhan, Hubei Province which was ultimately identified as a novel virus named SARS-COV-2 resulting in the disease named "coronavirus disease 2019", also known as COVID-19. On 13 January 2020, officials confirmed the first recorded COVID-19 case outside of China. After the disease started spreading all over the world, the World Health Organization (WHO) categorized this epidemic as a pandemic on 11 March 2020 [1]. As of 24 August 2020, COVID-19 has spread over 213 countries and territories around the world, resulting in over 23,586,023 reported cases of confirmed contamination and 812,527 deaths [2,3]. Figure 1 illustrates COVID-19 spreading over time, which is very alarming as the rate of the new victims is rising at a significantly higher rate. Some of the countries, such as 
Australia, that experienced a decreasing number due to social distancing, are also facing a second wave of the COVID-19 pandemic.

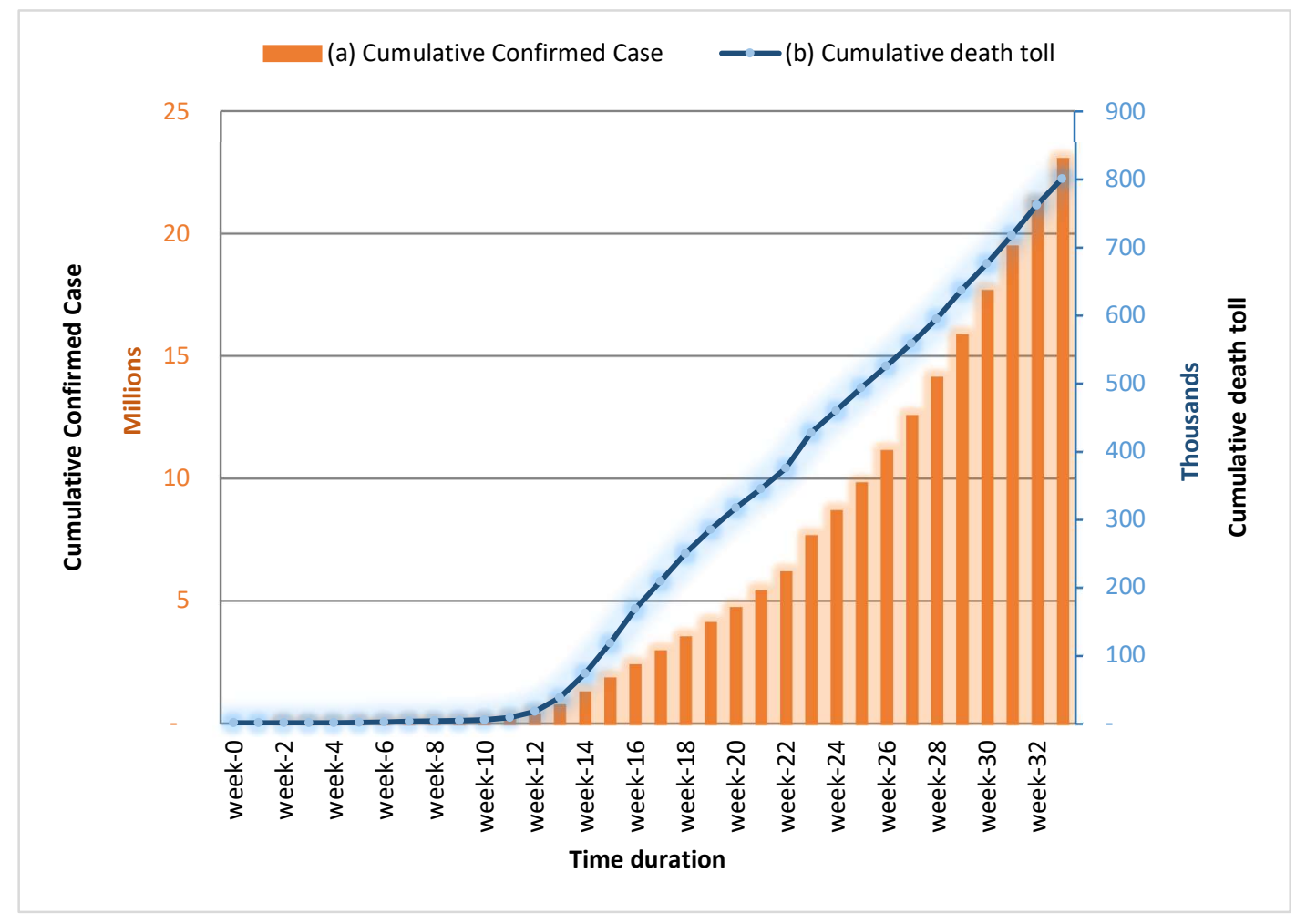

Figure 1. The statistics of the coronavirus disease 2019 (COVID-19) disease outbreak over the world, the figure shows (a) the accumulative figure of confirmed infected cases and (b) the accumulative figure of deaths.

The healthcare sector has put a great effort into COVID-19 diagnosis, screening and treating the infected individuals to address this pandemic. Governments and international organizations are aiming to organize an appropriate level of healthcare to mitigate the disease. To date, no medicine or vaccine has been invented to encounter this disease with complete assurance. So, different treatment methods and different medicines are applied as appropriate to remedy against this disease. On the other hand, authorities are imposing measures such as social distancing, area lockdown, the use of proper sanitization, etc. to prevent the further spreading of this pandemic. Therefore, to prevent the spreading of this disease, a collective effort is needed from all social entities such as the international organizations, governments, healthcare systems, and more essentially from the community. Moreover, the widespread integration of prospective technologies with effective healthcare management and resilient governance will reinforce the capacity to protect the society from COVID-19 disease [4].

With the emergence of intelligent technologies in this age of information from the global context, advanced artificial intelligence (AI) techniques promise massive applications in a variety of sectors. Figure 2 exhibits artificial intelligence and its subsections, especially from a machine learning (ML) point of view [4]. Although social distancing and the use of masks/gloves have been proven effective to slow down the spread, effective countermeasures and defense strategies need to be developed to build resilience against the pandemic through better monitoring and control. AI and ML, which have been successfully applied in a wide range of research areas, may have numerous prospective applications for achieving protection from COVID-19 disease. AI and ML can play a significant role in addressing the healthcare and social sector challenges caused by the COVID-19 pandemic. On a molecular level, AI can be utilized to approximate the construction of SARS-COV-2 associated proteins, classify present medications that can possibly be used as a remedy for the disease, suggest novel composites that 
can possibly contribute to the medicine development, design the potential objectives of the vaccine, and develop identification procedures as well as better realization of the virus contamination and severity level. From a medical viewpoint, AI can help improve the COVID-19 identification by analyzing medical imaging, offer different methods to study disease progression utilizing appropriate tools, and produce forecasts on infection spread based on compound statistical feedback, including digital medical history. From a societal standpoint, AI can be utilized for epidemiological exploration modeling, experiential statistics, including predicting the number of cases given different public policy choices. Sharing and presenting research ideas, along with extracting insights from statistics and simulations, are vital to speed up the response against the pandemic [5]. In such cases, the Internet of Things (IoT) can play a major role. AI coupled with IoT is more powerful in terms of the intelligent decision making and can help fight against this pandemic.

The Allen Institute for Artificial Intelligence is now sharing a digital library of articles that provides free access to COVID-19 data [6]. This database presently comprises over 44,000 articles; among them, 29,000 are full-text journals. Conjoining this database with other raw statistics from web links or social media can reveal further information and insights about the early detection and control of future outbreaks of SARS-COV-2 virus. Outbreak Response Management and Analysis System (SORMAS) and HealthMap are examples of surveillance-mapping tools that work online and allow early detection of contagious diseases in contrast to commonly used epidemiological methods. At this time, SORMAS and HealthMap are utilized for the observation of the COVID-19 situation [7]. On the other hand, The British Society of Thoracic Imaging (BSTI) and Cimar UK's Imaging Cloud Technology [8] have deployed an easy to access, open for all encrypted web platform to share imaging of patients identified or suspected to have COVID-19 [9]. The BSTI is creating a database containing imaging of confirmed patients with COVID-19 from these uploaded cases for further research. The goal is to broadcast new medical and investigative guidance quickly to medical service providers all over the country, utilizing these actual imaging samples [9].

Artificial Intelligence (AI) It is a system that is capable of acquiring data and information, analyse and learn from those data and apply
those learning to achieve certain requirement.

Machine Learning (ML)

It is a sub-section of artificial intelligence (AI) that is a system with facility to automatically learn and improve from experience without being explicitly encoded. Machine learning emphasizes on the improvement of computer programs which make them able to access data and utilize it for learning.

Supervised Learning is the method of training and testing in which, computers are trained with some old or predefined data sample and after that they utilize the experience to predict the new data samples.

Unsupervised Learning is the method of learning from given or acquired information without any administration of it and it continuously learns from the newly acquired statistics.

Reinforcement learning is the sub-section of machine learning concerned with how software agents map situations to actions in order to maximize the numerical rewards.

Figure 2. The relation between artificial intelligence and its components [10]. 
From the above discussion, we can summarize that organizations and researchers across different countries are putting their best effort to build resilience against this pandemic. As AI, ML, and IoT have been proven effective in a wide range of application areas, this paper concentrates on evaluating the scope of these emerging technologies and recent progress in developing the countermeasures against COVID-19 pandemic. Specifically, this paper addresses the following key research questions:

i. What are the emerging applications of $\mathrm{AI}$ and ML that can help toward building resilience against the COVID-19 pandemic? The answer of this question has been explored in Section 2.

ii. What are the social and information science-related applications of AI and ML that played an important role during this crisis? The answer of this question has been elaborated in Section 3.

iii. What are the roles of emerging technologies such as IoT for COVID-19 control and prevention? The answer of this question has been identified in Section 4.

iv. Is there any potential risk due to the integration of emerging intelligent techniques such as AI, ML, and IoT? The answer to this question has been explored in Section 5.

\section{Organization and Contents of the Paper}

In this paper, we summarize the impact of the emerging AI and ML technologies basically from two perspectives: (i) medical service-related applications and (ii) social science-related applications, as discussed in Sections 2 and 3, respectively. In Section 2.1, we provide insights on how medical imaging using AI and ML can help identify the presence of COVID-19 disease symptoms. Patient condition monitoring using AI and ML-based data analytics is presented in Section 2.2. In Section 2.3, we discuss how drug development and delivery can be automated and effectively performed using these technologies. Virus propagation modeling using AI and ML has been focused in Section 2.4. Based on the literature review, we highlight that the early prediction and detection can be beneficial to slow down the progress of this pandemic in Section 2.5. The medical sector has experienced that healthcare workers are highly vulnerable to COVID-19 disease, and therefore, in Section 2.6, we summarize some possible AI and ML-driven countermeasures to protect the valuable personnel who are contributing significantly to help fight against the pandemic.

Social and Information Science-related applications of AI and ML are presented in Section 3. Effective forecasting and prediction based on the COVID-19 statistics can help the society and nation to build important strategies and public policies. Section 3.1 discusses how AI and ML can be leveraged to help predict COVID-19 statistics. These emerging tools and techniques can also identify the influential service provider of the healthcare sector, as discussed in Section 3.2. These intelligent techniques can also help in measuring and deploying social distancing, as summarized in Section 3.3. To achieve protection against this pandemic, data availability can bring significant benefits. AI and ML-based applications can help in advanced data sharing, hosting, and analytics (presented in Section 3.4). AI governance is discussed in Section 3.5, and finally, Section 3.6 presents AI and ML-based social awareness improvement capabilities.

IoT-based applications gained its importance for COVID-19 patient identification and tracking. For example, contact tracing applications have helped to slow down the spread of the pandemic. Section 4 discusses the existing IoT-based COVID tracing mechanisms, as well as IoT-based drone applications, wearable technologies, telemedicine, etc., to demonstrate how IoT can help build resilience against the pandemic. Although AI, ML, and IoT have enormous potentials and prospects, misuses of these technologies can be very risky and cause significant loss. Therefore, potential risk factors and some new technologies to address these risks more effectively are also discussed in Section 5. Finally, the paper concludes with some brief remarks in Section 6.

\section{Application of AI and ML for the Medical Sector}

$\mathrm{AI}$ and ML in the medical sector have a broad range of applications, including the prediction and classification of statistical information. In particular, BlueDot Toronto, which was developed 
by infectious disease scientists to research innovative solutions for mitigating the initial SARS outbreak, designed the first risk-based solution for detecting the outbreak of SARS-COV-2 virus [11]. This successful demonstration of AI and ML in predicting disease outbreaks applied BlueDot's prior research on the SARS outbreak to implement advanced technologies. A report stated that BlueDot employed a disease-monitoring framework that scrutinized over 100,000 online documents globally in 65 languages every $15 \mathrm{~min}$ [4]. This system identified unusual statistics of pneumonia cases with unidentified cause in Wuhan, the ninth most populated city in China, and it warned about the occurrence of the disease considerably earlier than when it was formally recognized as COVID-19 [4]. Therefore, these advanced technologies can forecast such epidemics and create awareness among people to take the required pre-cautions for encountering the outbreaks.

Artificial intelligence application in detecting infectious diseases is extremely valuable in the medical sector and can bring revolutions to the healthcare practices. Integrating AI in the imaging processes has received significant attention within the healthcare sector. Machine-learning prototypes can scrutinize the medical images to identify the sickness at an initial phase. Such prototypes are driven by big data and deep learning algorithms to achieve the particular task. The prospective sectors where this image-oriented learning is possibly applied are pathology, ophthalmology, radiology, and dermatology [4]. The mitigation of diseases such as COVID-19 massively depends on the screening of people through pathogenic testing, which is a time-consuming process, and hence, precision is a must. In a study, the author introduced a medical identification process for COVID-19 based on radiographic variations in computerized tomography (CT) scans by implementing the deep learning process, which achieve $85.2 \%$ accuracy in the testing and validation stage. [4].

Machine learning can assist the work of medical professionals by analyzing and organizing an enormous amount of patient information stored in digital medical records. Moreover, Machine Learning is applied in different medical applications that include detecting patients with severe conditions who urgently require intensive care unit (ICU) facilities, identifying early symptoms of diseases, understanding the breathing condition of the patient by analyzing chest X-rays, etc. Therefore, $\mathrm{AI}$ and ML improves the performance of identification and prediction process and how administrative decisions are made in the medical sector [4]. In these circumstances of the COVID-19 pandemic, the aforementioned technologies have already aided medical professionals encountering the situations in a significantly effective manner.

\subsection{Medical Imaging for COVID-19 Patients}

Medical imaging such as CT scan and X-rays are widely used for diagnostic and treatment purposes. During the COVID-19 pandemic, the role of CT scan and X-ray imaging are very important for SARS-COV-2 virus detection. For this purpose, the emerging technologies such as AI and ML reinforce the imaging analysis by intelligent detection and classification of the abnormalities. For instance, AI-enabled disease type classification will help toward automation of the screening process, and this automatic process will reduce human interaction among the doctors and the patients. Thus, it acts as a safeguard to the medical imaging professionals and helps to reduce the spread of the virus. Furthermore, the computer-assisted AI-enabled automated classification and recommender systems support the radiologists to make clinical judgments, i.e., infection identification, tracing, projection with better accuracy, and efficiency [12].

The Reverse Transcription-Polymerase Chain Reaction (RT-PCR) test is typically used to measure the amount of any particular type of RNA. This RT-PCR is being used for the COVID-19 infection diagnosis [13]. However, the RT-PCR test has still some limitations. For example, as reported in [13], this powerful test produces high specificity, but the sensitivity from the test is very low (for example, as low as 59\%). Therefore, the laboratory assessment suffers from inadequate sensitivity, which needs to be improved to enhance the performance further and for wider adoption.

To solve the aforementioned problem related to the false positive diagnosis, the latest AI-empowered tools and techniques need to contribute significantly toward building resiliency 
against the COVID-19 pandemic. The success of the AI-enabled tools are not only limited as a prototype these days, as a good number of commercial applications have also been introduced. Those applications incorporate the strengths of powerful deep learning and intelligent techniques to help slow down the spread and improved identification of the virus and thus exhibit technological competence against the spread of the pandemic [12].

In [14], the authors describe a process where deep transfer learning was used with Generative Adversarial Network (GAN) for COVID-19 recognition in X-ray images of a patient's chest. Ian Goodfellow introduced the Generative Adversarial Network (GAN) in 2014, which is a type of deep learning algorithm. GAN prototypes are mainly comprised of two networks, generative and discriminative network. The generator network is designed to generate new fake data instances, which are similar to training data. The purpose of the discriminator is to differentiate between actual data and fake data generated from a generator network. The generator network produces fake data to evade the network, and the discriminator network identifies the difference between actual and fake data and tries to prevent the evasion, which is the mission of the GAN models. The shortage of records regarding COVID-19 such as chest X-rays images is the key motivation for considering this type of model in [14]. The main idea of this GAN-based study is to gather all the available imaging data and COVID-19 test results and then utilize the GAN network to create more datasets to assist in the identification of the virus from the available X-rays scans with the maximum accuracy.

Another study [15] presents an early detection of COVID-19 using machine learning techniques, where the detection procedure was employed on CT scans of an abdomen. The experienced radiologists identified from CT scans that COVID-19 indicates dissimilar characteristics as compared to other viral pneumonia. Hence, the medical specialists can identify the COVİD-19 infection in the initial stage. For investigations regarding the identification of COVID-19, four dissimilar datasets were designed by considering patches with dimensions as $64 \times 64,48 \times 48,32 \times 32$ and $16 \times 16$ from a number of $150 \mathrm{CT}$ scans. To improve the classification performance, a feature extraction process was applied to patches, which utilized algorithms such as Grey Level Co-occurrence Matrix (GLCM), Local Directional Pattern (LDP), Grey Level Run Length Matrix (GLRLM), Grey-Level Size Zone Matrix (GLSZM), and Discrete Wavelet Transform (DWT). Support Vector Machines (SVM) were used to categorize the extracted features. Ten-fold, 5-fold, and 2-fold cross-validations were applied throughout the categorization process. Precision, accuracy, specificity, sensitivity, and F-score metrics were utilized to assess the categorization performance. The best accuracies were achieved as $99.68 \%$ with 10 -fold cross-validation and the GLSZM feature extraction technique.

It is obvious that an initial identification of COVID-19 is essential to slow down the spread of COVID-19 and avoid transmission by an early quarantine of victims as well as tracing and isolation of close acquaintances. For patients diagnosed with COVID-19, the precise monitoring of disease development is a vital element of disease administration. Medical imaging records such as chest X-ray and CT scans play a significant part in confirming the positive identification of COVID-19 disease as well as observing the evolution of the disease. These sorts of imaging data exhibit irregular distinctive patterns that emerge right after the COVID-19 infection starts. These anomalies peaked during 6th to 11th day of the illness. The next most predominant pattern of lung conditions anomalies peaks during the 12 th to 17 th day of the illness [16]. Computer-Aided Diagnosis (CAD) methods that integrate X-ray and CT scan results with deep learning algorithms can help physicians as identification aids for COVID-19 and assist further to provide a better understanding of the disease progression.

\subsection{Patient Condition Monitoring Using Clinical Data}

The patient's condition can be monitored remotely by taking a patient's status or clinical data through the remote monitoring technology [7]. These technologies can link wirelessly to the networks via WiFi, Bluetooth, or cellular connection. The same idea can be implemented for the monitoring of infectious diseases such as COVID-19. This distant observing technique can be utilized to screen persons exposed to COVID-19 as well as close acquaintances of the infected person. These technologies 
can also be applied to prevent the exposure of medical professionals to the high-risk patient populations. In principle, the same technological arrangements presently utilized by remote monitoring programs can also be utilized to incorporate a temperature measurement tool such as a smart thermometer for monitoring individuals that are suspected of having COVID-19 [7]. AI and ML can significantly help to improve monitoring and identify abnormal patterns or behaviors. ML and AI-based advanced data analytics and signal processing can help to filter the data and identify the patterns.

\subsection{Drug Development, Selection and Delivery}

In [17], the authors use a drug-target interaction prototype titled as Molecule TransformerDrugTarget Interaction (MT-DTI) that uses the deep learning method to identify drugs that are available in the market to react on viral proteins of SARS-COV-2. In another paper [18], the authors formulated a model that uses machine learning technology to forecast the potential inhibitory synthetic antibodies for SARS-COV-2 virus. They gathered antibody sequences of the 1933 virus and their clinical patient neutralization reaction and pre-trained a machine learning model to forecast the antibody reaction. Utilizing graphical representation with different ML techniques, the authors selected thousands of antibody sequences and found eight steady antibodies that hypothetically inhibit SARS-COV-2. The authors combined bioinformatics, structural biology, and Molecular Dynamics (MD) simulations to authenticate the constancy of the candidate antibodies that can inhibit the SARS-COV-2 virus.

Nowadays, we are observing rapid advancement in every aspect of science and technology, and it is also the same scenario in the sector of biological science, where complex biological data such as DNA structures and protein sequences are now available. With the help of machine learning techniques, those available data can predict complex biological scenario to analyze the virus protein structure and potential antibodies. With proper and sufficient training data, a machine learning mechanism can be utilized to create relations between the virus entity and reaction of its probable antibody. A pre-trained ML tool can predict potential antibodies for a given protein sequence of a virus faster than the human immune system, which can be very useful while combating against any pandemic such as COVID-19 and can save many lives in a timely manner.

According to another study [4], in China, one approach that was utilized to overcome the medicine delivery concerns during the epidemic was by employing an IoT-based service platform (Figure 3 exhibits the workflow). The platform created orders by automatically analyzing the information from historical records. Those orders may be modified and submitted by the pharmacists and then sent to the medicine suppliers from where the medicines are distributed within a specified time. This had lessened the spread of the disease during medicine purchase and also saved resources and labor cost.

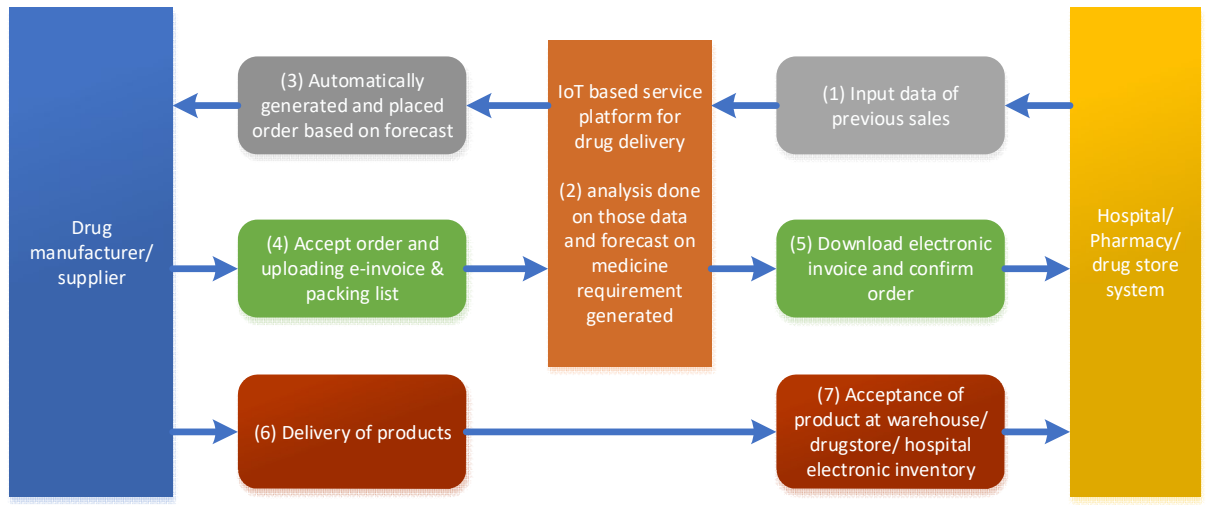

Figure 3. The Internet of Things (IoT)-based drug delivery system [4].

\subsection{Virus Propagation Modeling and Prediction}

The rapid spreading of the COVID-19 disease may be because of various reasons. One reason is the lack of information transparency at the initial phase of this pandemic outbreak. Sharing the 
pandemic-related information in a timely and precise way is tremendously vital for the anti-epidemic reaction of the community. The transparency in information sharing could have minimized the spread of this disease at the initial phase of the pandemic. Another reason is the unavailability of a proper systematic diagnosis standard for this virus. The prompt development of testing systems for a novel virus is very challenging. The symptoms of this virus are highly alike to symptoms of other less dangerous flu, which worsens the accuracy of diagnosis. Finally, due to the absence of a proper epidemic warning and prediction arrangement, we lost the chance to prohibit the spreading of this disease at the early phase.

In the current SARS-COV-2 transmission situation, the epidemiological study is tremendously essential for preventing the transmission by tracing the contagious trails and detecting the connection chains contributing to the rapid spreading. However, the outbreak of the COVID-19 was boosted up by the high movement of people during the most significant customary carnival of China, which worsened the transmission of the virus and at the same time greatly amplified the challenges of the epidemiological investigation [19]. It is vital to observe and predict the evolution of an epidemic while making decision against this kind of health crisis. In this situation, the mathematical propagation model has achieved more consideration and attention in terms of the epidemiological research. The Susceptible-Infected-Removed (SIR) model is one of those mathematical propagation models. The research on plague in the early 20th century introduced this SIR model. Remarkable development has been made in the mathematical propagation model of epidemiological research since the middle of the 20th century. At present, some important factors inducing the epidemic transmission were incorporated in the classic SIR model, such as the model taking into consideration the maturation phase, the SEIRS (Susceptible-Exposed-Infectious-Recovered-Susceptible) model taking into consideration the age of people and the population exposed to an epidemic and the Susceptible-Infectious-Susceptible (SIS) model including the birth and death of vulnerable patients. Some dynamical prototypes were planned to consider only specific disease analysis. For instance, the dynamical models were designed to simulate the spreading of human immunodeficiency virus (HIV), severe acute respiratory syndrome (SARS), and Middle East Respiratory Syndrome (MERS). With the advancement of innovative techniques, complex network theories and machine learning techniques were incorporated with the mathematical propagation model, which generated an effective method of epidemic prediction.

The construction of the SIR prototype for an epidemic analysis requires defining a number of vital parameters (such as the rate of infection and the rate of infection elimination) factually or statistically. The SIR prototype has established a complex model enabling a more detailed classification of individuals - for example, the exposed group and the protection group. As a matter of fact, the more accurate the prototype, the more chances are there that it can make an accurate forecast. However, for better accuracy, the model requires more realistic data to define additional factors. Alternately, some effort has also been made for developing a simplified prototype. For instance, the renowned logistic equation was used as the two sections "S-I" (Susceptible-Infectious) prototype, which can be additionally incorporated for some procedures in the modeling of SARS. The aforementioned studies demonstrate that the SIR-family prototypes can capture the base mechanism of the epidemic transmission at diverse complex stages quite well.

In one study [19], the authors tried to represent an initial forecast of the COVID-19 epidemic obtained from a basic SIR prototype. The justification of the obtained epidemiological information was investigated initially to achieve a realistic approximation of the fundamental factor, such as the rate of infection. Focusing on the rate of infection and the rate of disease elimination, a number of trials were planned to simulate the transmission of SARS-COV-2 virus under diverse stages of anti-epidemic measure and precautions. The forecasted infection intervals for the collective cases and the fading-out time of the epidemic are presented. The outcomes of the study are supposed to deliver vital statistics for crisis management against this COVID-19 pandemic. 


\subsection{Early Prediction or Detection of COVID-19 Syndromes}

AI plays a significant role in identifying the collection of cases and forecasting the location where this disease will spread in the future by gathering and scrutinizing all previous data related to this outbreak. This technology can detect the disease characteristics and can predict its nature from the obtained information through social media and other digital or paper media platforms, regarding the possibilities of the contagion and potential nature of spreading. Additionally, it can predict the number of infected cases and death in any specific area based on related data and information [20].

In [21], the authors present a machine learning analysis of the COVID-19 pandemic based on the information of the initial phase of infection dynamics. The objective is to extract actionable insights for the improved understanding of community health. These understandings include the disease transmission probability, the rate at which mild infection transforms into a serious infection, asymptomatic approximations for infections, and forecasts of new infections over time.

In a study [22], the authors demonstrate the Bayesian Convolutional Neural Networks (BCNN), which is based on Monte-Carlo Dropout to determine the uncertainty in the methods of deep learning techniques that are used to improve the performance of the detection process while investigating chest X-ray reports of COVID-19 victims. They conclude that if the model is responsive about the system uncertainty, this method will improve the performance hugely and can facilitate the medical sector with a wider implementation of advanced technologies such as artificial intelligence.

In [23], the authors proposed a machine learning method-assisted SIR model to investigate the COVID-19 epidemic in a practical scenario. In this effort, they utilize the traditional Kermack-Mckendrick SIR prototype to describe the spreading pattern of the epidemic of COVID-19. The SIR prototype can model how an infection is transmitted over a population. In the research, they consider the total number of the population as a constant and classified them into different categories for more accuracy.

In [24], the authors utilize an effective approach to identify potential therapeutic molecules that combined machine learning technique and highly reliable docking simulation models. The researchers initially apply the screening plan to two drug datasets to discover a number of therapeutic molecules that have a strong bindness to the systems. The authors use the ML model to screen a huge number of bio-particle datasets to create a list of possibly beneficial composites with high rank and order for more confirmation. Overall, this effort will enhance the understanding of molecular treatment of COVID-19 and at the same time deliver an effective way of drug selection by merging high-performance machine learning prototypes with high-fidelity models for speeding up the therapeutic treatment of the diseases. A data-driven model trained with both data and first principles is proposed in [25]. The model can quickly be re-trained any time when new data become available.

\subsection{Protecting Healthcare Workers}

Most researchers have identified that artificial intelligence has brought an exemplary change in the medical sector and there might be value in the use of AI to the present COVID-19 epidemic, for instance, in forecasting the place of the next occurrence. As David Heymann (Assistant Director, WHO) headed the global response to SARS, he clarifies some vital factors that are essential for an effective public health response to an occurrence of a novel contamination. These factors include a consideration of transmissibility and vulnerable groups; forming the natural history of contamination, comprising the development period and death rate; recognizing and characterizing the underlying reasons; and, in some cases, epidemiological modeling to propose effective prevention and control measures [26]. These data can be gathered from those employed at epidemic locations virtually linked with the WHO. This approach functioned for SARS and is, again, a major source of information for COVID-19, as discussed by David Heymann. Finally, this information can be utilized to train and introduce the AI application for its dedicated task. The role of the healthcare professionals is important. They are not only helping to collect the COVID data but also helping the vulnerable patients in person, risking their own life. AI and ML can help identify the risk and also help automate a number of functionalities. 
Reflecting on a swiftly rising fear in the present epidemic that concerns the contamination of medical staff, Kuan Chen, founder of Infervision, points out that the AI application can assist in protecting the healthcare professionals [26]. Similarly, researchers and vendors are trying to utilize AI-enabled medical devices to reduce direct contact during SARS-COV-2 virus diagnosis and treatment.

\subsection{AI-Enabled Self-Testing Framework}

An AI-enabled self-testing framework is shown in Figure 4. The framework has three major phases. At the initial phase, the patient-side sensor devices will collect the specimen supplied by the patient. Based on the samples collected by the sensors, AI and ML-based analytics will be used for further processing. Decisions can be made locally in an edge node or could be in the cloud when compared with historical data if necessary. Finally, based on the test results, patients will be notified via mobile phone. To communicate among the peers, the blockchain-based framework could be very helpful. As the test results are very sensitive and require better security and privacy protection, blockchain may serve that purpose. Being a distributed ledger technology, the blockchain-based framework can be connected with local and international databases, while the AI-based decision-making process helps patients with self-diagnosis [27].

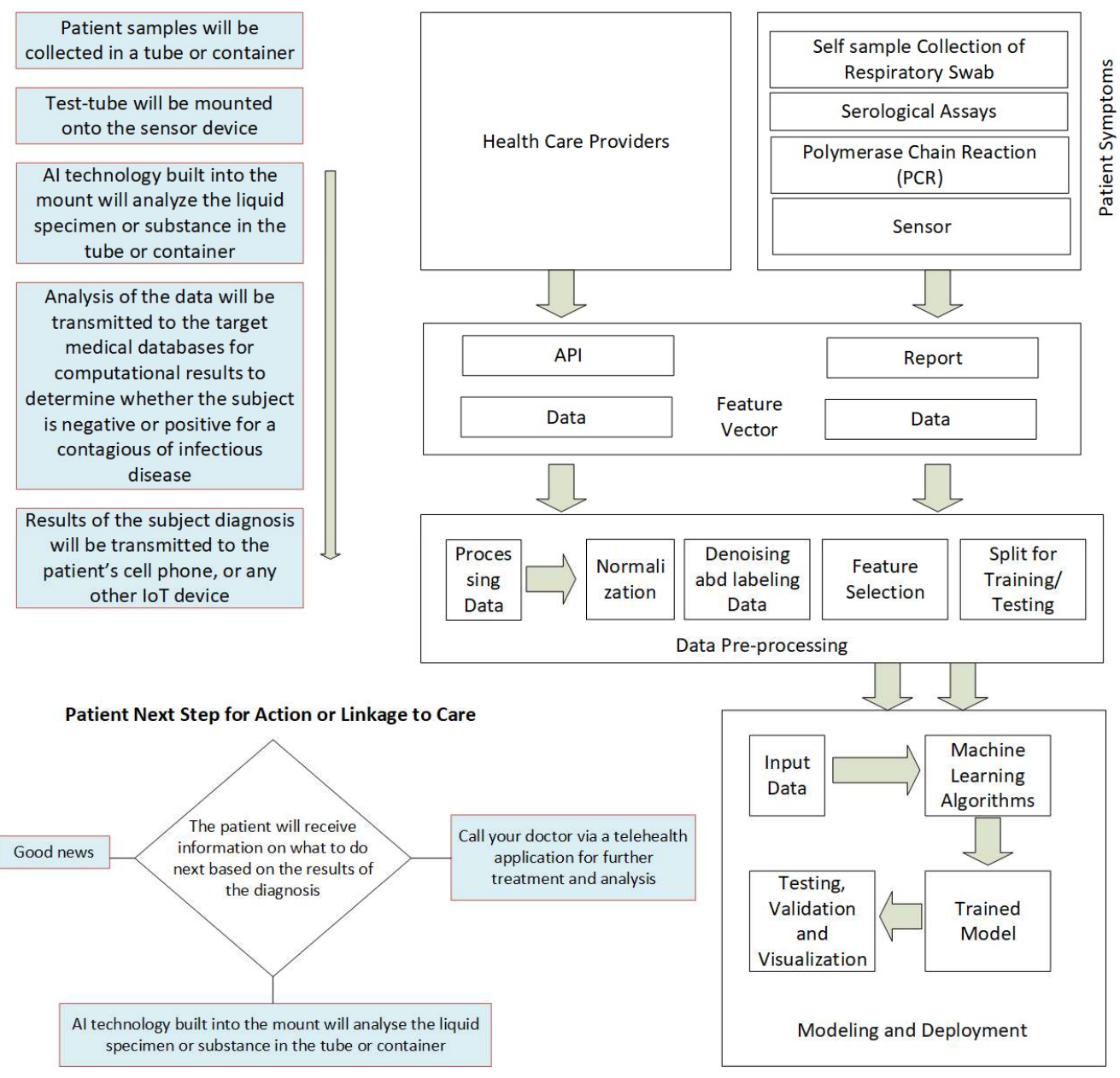

Figure 4. Artificial intelligence (AI)-enabled self-testing framework, adopted from [27].

Table 1 summarizes the articles and projects related to the AI applications toward improved resiliency against the COVID-19 pandemic. 
Table 1. Summary of the research contributions considering artificial intelligence (AI) to mitigate COVID-19.

\begin{tabular}{|c|c|c|c|}
\hline Paper/Project Name & Reference No. & Domain & Contribution \\
\hline $\begin{array}{l}\text { Madurai Elavarasan, R. and Pugazhendhi, } \\
\text { R. (2020). Restructured society and } \\
\text { environment: A review on potential } \\
\text { technological strategies to control the } \\
\text { COVID-19 pandemic. }\end{array}$ & [4] & $\begin{array}{l}\text { Drug development, selection, } \\
\text { and delivery }\end{array}$ & $\begin{array}{l}\text { Review of potential technologies } \\
\text { such as AI, ML, and IoT in the } \\
\text { healthcare sector for forecast of the } \\
\text { epidemic, disease investigation, } \\
\text { medicine delivery, etc. }\end{array}$ \\
\hline $\begin{array}{l}\text { Alwashmi, M. F. (2020). The Use of Digital } \\
\text { Health in the Detection and Management } \\
\text { of COVID-19. }\end{array}$ & [7] & $\begin{array}{l}\text { Patient condition monitoring } \\
\text { using clinical data }\end{array}$ & $\begin{array}{l}\text { Review of outbreak response } \\
\text { management and analysis system } \\
\text { and remote monitoring } \\
\text { technology for infected victims. }\end{array}$ \\
\hline $\begin{array}{l}\text { Shi, F., Wang, J., Shi, J., Wu, Z., Wang, Q., } \\
\text { Tang, Z., Shen, D. (2020). Review of } \\
\text { Artificial Intelligence Techniques in } \\
\text { Imaging Data Acquisition, Segmentation } \\
\text { and Diagnosis for COVID-19. }\end{array}$ & [12] & $\begin{array}{l}\text { Medical Imaging for } \\
\text { COVID-19 patients }\end{array}$ & $\begin{array}{l}\text { Review of integration of artificial } \\
\text { intelligence in imaging for } \\
\text { detection, diagnostics, and tracing } \\
\text { the spread of the epidemic. }\end{array}$ \\
\hline $\begin{array}{l}\text { Meng Li. Review: Chest CT features and } \\
\text { their role in COVID-19. Radiology of } \\
\text { Infectious Diseases, www.sciencedirect.com }\end{array}$ & [13] & $\begin{array}{l}\text { Medical Imaging for } \\
\text { COVID-19 patients }\end{array}$ & $\begin{array}{l}\text { Review of COVID-19 disease } \\
\text { detection by imaging tools such as } \\
\text { CT scans. }\end{array}$ \\
\hline $\begin{array}{l}\text { Mohamed Loey, Florentin Smarandache } \\
\text { and Nour Eldeen M. Khalifa. Within the } \\
\text { Lack of Chest COVID-19 X-ray Dataset: A } \\
\text { Novel Detection Model Based on GAN and } \\
\text { Deep Transfer Learning. }\end{array}$ & [14] & $\begin{array}{l}\text { Medical Imaging for } \\
\text { COVID-19 patients }\end{array}$ & $\begin{array}{l}\text { Describes a deep learning process } \\
\text { that used Generative Adversarial } \\
\text { Network (GAN) for COVID-19 } \\
\text { investigation in chest X-ray } \\
\text { images of patients. }\end{array}$ \\
\hline $\begin{array}{l}\text { Mucahid Barstugan, Umut Ozkaya, Saban } \\
\text { Ozturk. "Coronavirus (COVID-19) } \\
\text { Classification using CT Images by Machine } \\
\text { Learning Methods". }\end{array}$ & [15] & $\begin{array}{l}\text { Medical Imaging for } \\
\text { COVID-19 patients }\end{array}$ & $\begin{array}{l}\text { Describes the implementation of } \\
\text { the machine learning technique in } \\
\text { the process of COVID-19 } \\
\text { disease detection. }\end{array}$ \\
\hline
\end{tabular}

Sara Hosseinzadeh Kassania, Peyman

Hosseinzadeh Kassasnib, Michal J.

Wesolowskic, Kevin A. Schneidera, Ralph

Deters. "Automatic Detection of

16] Medical Imaging for COVID-19 patients

Reviews the implementation of

Coronavirus Disease (COVID-19) in X-ray

and CT Images: A Machine Learning

Based Approach".

[16] COVID-19 patients

machine learning methods to identify COVID-19 disease in medical images.

Beck, B. R., Shin, B., Choi, Y., Park, S., and

Kang, K. (2020). Predicting commercially available antiviral drugs that may act on the novel coronavirus (SARS-CoV-2) through a

drug-target interaction deep

learning model.

Rishikesh Magar, Prakarsh Yadav and Amir

Barati Farimani. "Potential Neutralizing

Antibodies Discovered for Novel Corona

Virus Using Machine Learning."

Zhong, L., Mu, L., Li, J., Wang, J., Yin, Z., and Liu, D. (2020). Early Prediction of the 2019 Novel Coronavirus Outbreak in the

Mainland China based on a Simple Mathematical Model.

Vaishya, R., Javaid, M., Khan, I. H., and Haleem, A. (2020). Artificial Intelligence (AI) applications for COVID-19 pandemic.

Malik Magdon-Ismail. “Machine Learning the Phenomenology of COVID-19 From Early Infection Dynamics".

Early prediction or detection of COVID-19 syndromes

Virus propagation modeling and prediction

Drug development, selection, and delivery

Drug development, selection, Implementation of a deep learning method to identify potential medicine against COVID-19 disease among commercially available medicine.

Application of artificial intelligence to forecast potential antibodies against SARS-COV-2 virus.

Describes early prediction of COVID-19 epidemic based on SIR epidemic prediction model.

Review of the application of artificial intelligence technologies in prediction of COVID-19 spreading

Present implementation of machine learning analysis on COVID-19 epidemical statistics to forecast disease transmission.

Demonstration of Monte-Carlo Dropweight-based Bayesian

Biraja Ghoshal and Allan Tucker

"Estimating Uncertainty and

[22] Early prediction or detection Convolutional Neural Networks

Interpretability in Deep Learning for of COVID-19 syndromes

method to improve the performance of COVID-19 detection process.

Babacar Mbaye Ndiaye, Lena Tendeng

Diaraf Seck. "Analysis of the COVID-19

pandemic by SIR model and machine

23] Early prediction or detection

Proposes an SIR model supported learning techniques for forecasting".
Early prediction or detection
of COVID-19 syndromes the epidemic in the rol westigate 
Table 1. Cont.

\begin{tabular}{|c|c|c|c|}
\hline Paper/Project Name & Reference No. & Domain & Contribution \\
\hline $\begin{array}{l}\text { Rohit Batra, Henry Chan, Ganesh Kamath, } \\
\text { Rampi Ramprasad, Mathew J. Cherukara, } \\
\text { and Subramanian Sankaranarayanan. } \\
\text { "Screening of Therapeutic Agents for } \\
\text { COVID-19 using Machine Learning and } \\
\text { Ensemble Docking Simulations". }\end{array}$ & [24] & $\begin{array}{l}\text { Early prediction or detection } \\
\text { of COVID-19 syndromes }\end{array}$ & $\begin{array}{l}\text { Proposes the utilization of a } \\
\text { machine learning and docking } \\
\text { simulation model to identify } \\
\text { therapeutic molecules. }\end{array}$ \\
\hline $\begin{array}{l}\text { Luca Magri and Nguyen Anh Khoa Doan. } \\
\text { "First-principles machine learning } \\
\text { modelling of COVID-19". }\end{array}$ & [25] & $\begin{array}{l}\text { Early prediction or detection } \\
\text { of COVID-19 syndromes }\end{array}$ & $\begin{array}{l}\text { Proposes a data-driven model that } \\
\text { is trained with both data and first } \\
\text { principles on an epidemiological } \\
\text { model. }\end{array}$ \\
\hline $\begin{array}{l}\text { Becky McCall. "COVID-19 and artificial } \\
\text { intelligence: protecting health-care workers } \\
\text { and curbing the spread". }\end{array}$ & [26] & Protecting healthcare workers & $\begin{array}{l}\text { Review of artificial intelligence } \\
\text { application in protecting } \\
\text { healthcare professionals. }\end{array}$ \\
\hline
\end{tabular}

\section{Social and Information Science-Related Applications Using AI and ML}

On social perspectives, AI has been used in numerous regions of epidemiological exploration modeling, including predicting the number of cases to develop different public policy choices as per the requirement and identifying the regions with different stages and different degrees of severity of the pandemic. Another interesting application of AI is measuring the social distancing, which will play a vital role while encountering this epidemic.

\subsection{Forecast the COVID-19 Statistics}

As we already discussed in the above section, an AI and ML-driven system can forecast the number of COVID-19-infected persons, which can help developing public policies to achieve protection against the pandemic. In [28], the authors demonstrate a new and well-timed approach that combines disease approximations from systematic prototypes with digital traces via interpretable machine-learning procedures to consistently predict COVID-19 activity in Chinese provinces in real time. That technique successfully produces precise and constant predictions 2 days earlier. The model utilizes information from the official medical records, internet search related to COVID-19, and daily forecast from the Global Epidemic and Mobility (GLEAM) model. The proposed machine-learning technique uses a bunching method that allows the manipulation of a three-dimensional figure of COVID-19 activity through the region of China, and a data augmentation procedure to deal with the inadequate amount of available past disease activity reports along with patterns of evolving epidemics. This prototype's ability to predict disease spread outperforms a number of standard prototypes in most of the provinces of China and could be readily applied to other geographical locations that are currently infected with the epidemic to assist the authorities in strategy development.

\subsection{Identify Influential Service Providers}

Recognizing the greatest influential providers in a medical sector is a vital stage for optimizing the utilization of accessible healthcare resources and confirming the more efficient distribution of matched healthcare services while building resilience against pandemics such as COVID-19. In [29], the authors offered a privacy-preserving network-based technique for detecting influential providers inside the medical sector. Initially, the authors assembled a provider-networking system by engaging the openly accessible data on the locations and categories of healthcare service providers. Next, the authors rank the nodes in the generated provider-networking system on the basis of the nodal influence metrics. Then, the authors assessed the influence of those top-ranked nodes in the provider-networking system by comparing network indicators and identified the most influential provider.

\subsection{Measuring Social Distancing}

The COVID-19 pandemic has quickly spread around the world and triggered changing guidelines to maintain physical distancing for slowing down the spread of the infection. In [30], the authors 
demonstrate a novel hybrid machine learning prototype, SIRNET (named after the foundational epidemiological model SIR), for predicting the transmission of the COVID-19 epidemic that combines with the epidemiological prototypes. The authors utilized the characterization of spatiotemporal cell-phone movement information, along with population density and other local data points. In that work, trial outcomes from SIRNET are considered to create initial boundaries on such localized movement that enable disease containment over time. The prototype can support studying non-pharmacological mitigation techniques that minimize corresponding cases and develop control mechanisms for a prolonged period of time.

SIRNET is a hybrid between machine learning methods, physical science, and epidemic modeling. The epidemic modeling generates significant variables from a physical perspective that adds a spontaneous understanding of how the prototype can be used for forecasting disease trends. In addition, machine learning methods offer an instrument for interpreting variables-for example, movement, non-pharmacological mitigation techniques, and population demographics-into variables that influence a pandemic prototype. It also allows us to determine connections between real-world trends on the spread of COVID-19, as well as the influence of underlying scenarios such as relaxing social distancing rules.

\subsection{Data Sharing and Hosting}

Nowadays, it is a common practice to share news regarding an ongoing incident in social media by the majority of the users. From the beginning of the COVID-19 pandemic, people have utilized social media to interchange different types of data about the disease at an increasing volume. To build resilience against this pandemic, only related and appropriate data are valuable for the community and administration. Hence, it is essential to classify the categories of data and to analyze the way it is being broadcast on social media so that proper data-sharing policies can be applied. This article in [31] utilizes Weibo data and natural language processing techniques to categorize seven types of COVID-19-related situational information. The results provide data-driven insights into information. The conclusion of this study also indicates that different strategies should be used while publishing different types of circumstantial news or information. These carefully chosen publishing strategies can also help the authorities control the huge amount of information regarding the COVID-19 situation. Additionally, this can help the researchers construct information-based emergency and crisis response plans.

\subsection{AI Governance}

The wide adoption of AI has impacted our socio-economic life, and therefore, it has a significant influence on government policy and decision making. In this regard, article [32] described the detailed effects of computerized decision support mechanisms on community services and the growing potentials for administrations to become an indispensable part of the digital community. The government authorities can compare the suitability of the prospective technologies while controlling and perhaps avoiding undesirable effects. This is highly important in the present situation of the COVID-19 epidemic where artificial intelligence and the supporting governing structure of the information network have become strategic concerns, as a large number of inventions are based on big data acquisition from smart appliances and the real-time availability of data and services.

The utilization of computerized decision-making systems in the public sector has certain values and requirements that it offers to deliver. This type of computerized system is important in the current situation of the COVID-19 pandemic, as the authorities are developing applications or tools that can monitor human mobility in an effort to reduce the probabilities of spreading.

For most of the artificial intelligence-based applications used in the public sector, the goal is to increase the efficiency and value of services, which is not completely addressed in the case of many other technology-agnostic services of the public sector. Moreover, it is beneficial to measure the appropriateness of the existing performance measurement tools and determine the way they can be further developed in the future, particularly if the condition after crisis does not bring us to "normality" 
and rather forces us collectively to alter our lifestyles and brings challenges to our regular social life and if the preventive actions are necessary to anticipate new challenges.

\subsection{Raising Social Awareness}

It is a great challenge to control the fast-growing "Infodemic" in the present situation of the COVID-19 pandemic. This involves enabling social awareness and a sensible behavior model in order to avoid and minimize occurrences such as the present pandemic. Thus, there is an ongoing effort to raise social awareness by providing up-to-date information and minimize the threat of false information. In [33], the authors exhibit an AI-enabled Infodemic Management solution WashKaro that utilizes natural language processing (NLP) methods, machine learning, and m-Health to reach out to the community, where audio-visual content used is translated in native languages. WashKaro provides reliable sources of data with regular bulletins, which is supplemented with a Bluetooth-based Contact Tracer, WHO directive-based Symptom Self-Assessment tool, and human-authenticated information delivered in native language.

To build resilience against the pandemic of COVID-19, it has become fundamentally essential to broadcast precise and well-timed data regarding the epidemic. As a result of the widespread internet connectivity and smart appliances usage, social sensing is developing as a dynamic sensing prototype to gather live opinions from online users. In another paper [34], the researchers demostrate CovidSens, the idea of social-sensing-oriented threat warning arrangements to inform the general public regarding the COVID-19 epidemic. The CovidSens idea is inspired by two of the latest observations: (i) people share their health status and COVID-19 experience actively on social media, and (ii) news agencies and official channels regarding COVID-19 news and warnings are relatively slower than the people's activity on social media regarding the pandemic. They anticipate an extraordinary prospect to leverage the posts created by the social media users to form a live analytics platform for collecting and broadcasting crucial data regarding the COVID-19 spreading. Precisely, the idea of CovidSens tries to answer the following questions: How to track the spread of COVID-19? How to filter reliable data regarding the disease with the existence of prevalent rumors and misrepresentation in social media? How to notify the general public about the up-to-date status of the spread timely and efficiently and prepare them for the circumstances? In the article, the authors demonstrate the roles of CovidSens and find the impending challenges in the implementation of dependable social-sensing-oriented threat notification systems. The authors suggest that the methods introduced by numerous disciplines (for instance, machine learning, estimation theory, constrained optimization) can be effective in addressing the challenges. Table 2 summarizes the articles related to the AI applications in social/information sciences for managing the pandemic.

Table 2. Summary of the research contributions considering AI for social science applications.

\begin{tabular}{|c|c|c|c|}
\hline Paper/Project Name & Reference No. & Domain & Contribution \\
\hline $\begin{array}{l}\text { Dianbo Liuy, Leonardo Clementey, } \\
\text { Canelle Poiriery, Xiyu Ding, Matteo } \\
\text { Chinazzi, Jessica T Davis, Alessandro } \\
\text { Vespignani, Mauricio Santillana. "A } \\
\text { machine learning methodology for } \\
\text { real-time forecasting of the 2019-2020 } \\
\text { COVID-19 outbreak using Internet } \\
\text { searches, news alerts, and estimates } \\
\text { from mechanistic models". }\end{array}$ & [28] & $\begin{array}{l}\text { Forecasting the } \\
\text { COVID-19 statistics }\end{array}$ & $\begin{array}{l}\text { Demonstrates the application of } \\
\text { machine learning methods to } \\
\text { forecast COVID- } 19 \text { transmission } \\
\text { based on internet data, news, and } \\
\text { epidemical forecast model. }\end{array}$ \\
\hline $\begin{array}{l}\text { Qi, X., Mei, G., Cuomo, S., and Xiao, L. } \\
\text { (2020). A network-based method with } \\
\text { privacy-preserving for identifying } \\
\text { influential providers in large } \\
\text { healthcare service systems. }\end{array}$ & [29] & $\begin{array}{l}\text { Identify influential } \\
\text { service provider }\end{array}$ & $\begin{array}{l}\text { Proposes a network-based privacy } \\
\text { preserving model to detect the } \\
\text { influential service provider of the } \\
\text { medical sector. }\end{array}$ \\
\hline
\end{tabular}


Table 2. Cont.

\begin{tabular}{|c|c|c|c|}
\hline Paper/Project Name & Reference No. & Domain & Contribution \\
\hline $\begin{array}{l}\text { Nicholas Soures, David Chambers, } \\
\text { Zachariah Carmichael, Anurag } \\
\text { Daram, Dimpy P. Shah, Kal Clark, } \\
\text { Lloyd Potter, Dhireesha Kudithipudi. } \\
\text { "SIRNET: Understanding Social } \\
\text { Distancing Measures with Hybrid } \\
\text { Neural Network Model for COVID-19 } \\
\text { Infectious Spread". }\end{array}$ & [30] & Measuring social distancing & $\begin{array}{l}\text { Demonstration of a unique } \\
\text { machine learning model } \\
\text { "SIRNET" to predict COVID-19 } \\
\text { spreading with the help of an } \\
\text { epidemiological model, SIR. }\end{array}$ \\
\hline $\begin{array}{l}\text { Li, L. et al. (2020). Characterizing the } \\
\text { Propagation of Situational } \\
\text { Information in Social Media During } \\
\text { COVID-19 Epidemic: A Case Study } \\
\text { on Weibo. }\end{array}$ & [31] & Data sharing and hosting & $\begin{array}{l}\text { Demonstrates methods to } \\
\text { categorize data and information } \\
\text { shared in social media related } \\
\text { to COVID- } 19 \text {. }\end{array}$ \\
\hline $\begin{array}{l}\text { Kuziemski, M. and Misuraca, G. } \\
\text { (2020). AI governance in the public } \\
\text { sector: Three tales from the frontiers } \\
\text { of automated decision-making in } \\
\text { democratic settings. }\end{array}$ & [32] & AI governance & $\begin{array}{l}\text { Describes the influence of artificial } \\
\text { intelligence on government policy } \\
\text { and decision making. }\end{array}$ \\
\hline $\begin{array}{l}\text { Rohan Pandey, Vaibhav Gautam, } \\
\text { Chirag Jain, Priyanka Syal, Himanshu } \\
\text { Sharma, Kanav Bhagat, Ridam Pal, } \\
\text { Lovedeep Singh Dhingra, Arushi, } \\
\text { Lajjaben Patel, Mudit Agarwal, } \\
\text { Samprati Agrawal, Manan Arora, } \\
\text { Bhavika Rana, Ponnurangam } \\
\text { Kumaraguru, Tavpritesh Sethi. "A } \\
\text { Machine Learning Application for } \\
\text { Raising WASH Awareness in the } \\
\text { Times of COVID-19 Pandemic". }\end{array}$ & [33] & Raising social awareness & $\begin{array}{l}\text { Demonstrates a social awareness } \\
\text { app "WashKaro" based on } \\
\text { artificial intelligence where } \\
\text { audio-visual content used is } \\
\text { translated in native languages. }\end{array}$ \\
\hline $\begin{array}{l}\text { Md Tahmid Rashid, Dong Wang. } \\
\text { "CovidSens: A Vision on Reliable } \\
\text { Social Sensing based Risk Alerting } \\
\text { Systems for COVID-19 Spread". }\end{array}$ & [34] & Raising social awareness & $\begin{array}{l}\text { Demonstrates a COVID-19 risk } \\
\text { alerting system that is based on } \\
\text { sensing information about the } \\
\text { epidemic on social media. }\end{array}$ \\
\hline
\end{tabular}

\section{IoT Applications during COVID-19}

With the advancement of information and communication technologies, IoT-based techniques have sparked a growing interest among healthcare applications developers. Undoubtedly, IoT applications emerge as a highly viable option for COVID-19 pandemic management. IoT technologies can be utilized for tracing the COVID-19 infection spread pattern, diagnosing COVID-19 patients, providing telemedicine services, and integrating these applications to wearables. In the following subsections, different IoT applications for COVID-19 disease management are outlined.

\subsection{Existing Contact Tracing Mechanisms}

IoT-based contact tracing applications have evolved as an effective solution for managing COVID-19 across different countries and regions [35-39]. The contact tracing mechanism requires citizens with COVID-19 to disclose their contact list to a trusted party such as public health officers. Then, based on the location of the tested citizen, other people who have been at the same location or had interactions with the person infected with COVID-19 are requested to take actions such as self-quarantine or testing [40]. Enabling IoT technologies for contact tracing can improve the efficiency in terms of automated processing, quick response, and most importantly, the privacy of the infected person [40]. There are a number of IoT-based contact tracing solutions proposed for the COVID-19 pandemic, as outlined in the following section.

Trace Together: This app has been released by the Singapore government for mobile applications in March 2020. The app uses Bluetooth technology to exchange randomly generated time-varying tokens among nearby phones as well as with the central server [41]. Upon installation of the app, a pseudonym will be generated and associated with the user ID or phone number. Then, the central server generates encrypted random tokens for each user, which the user broadcasts at pre-defined time intervals [42]. A person identified as COVID-19 positive will be requested to provide information of 
tokens received by his/her phone, which the government can decrypt and relate with phone numbers and identities [41].

Decentralized Privacy-Preserving Proximity Tracing (DP3T): This decentralized solution is proposed by the Pan-European Privacy-Preserving Proximity Tracing project team [43], which prompts individual users to generate daily random keys and broadcast ephemeral identifiers in random order. The users store all identifiers along with their proximities in the device database. If a user is detected as COVID-positive, the central health authority will request the user's secret key for the first day when the user became infected. Then, the secret key is then broadcast to all users, who can compute ephemeral identifiers for that specific day and subsequent days. As a result, other users can check if they were in close proximity of the user tested as COVID-19 positive for the past few days [42].

Efficient Privacy-Preserving Contact Tracing (EPIC): In this solution, the user devices exchange Bluetooth messages with nearby user devices and the server [44]. When the server has information on the infected users, it offers data-matching services only to users who are interested to know if they have shared the same location with the infected person. For this, the interested user sends timestamp messages to the server using homomorphic encryption, and the server reports back with the overlapped timestamps from the infected user. Then, the interested user sends the matched timestamps to the server, which computes the distance between the interested user and user infected with COVID-19 and forwards the information [42].

Contact Categorization: The solution utilizes contact categories rather than locations for infection spread detection. The user devices store a list of contacts based on the received Bluetooth messages. The developed application matches this list with the list of people infected with the virus, as obtained from the health authority. When a match is found, the application categorizes the contact based on the duration for which the non-infected user has been in contact with the user infected with COVID-19. The categorization helps the health authority prioritize testing when the infection spreading level is very high. Based on the signal strength, the proximity of the infected and non-infected users can also be obtained similar to the other approaches [45].

Privacy-Sensitive Protocols and Mechanisms for Mobile Contact Tracing (PACT): The solution enables users to generate pseudorandom IDs and broadcast to nearby users. Each user stores the received ID and time stamp. The users infected with COVID-19 upload the IDs and time stamps to the server, and the server adds the information to the list of users tested as COVID-positive. Any user can download this list and extract the IDs as well as time stamps to match with its local storage and find out if the user is in close proximity of the user infected with COVID-19. The solution allows for a time delay before the ID, and time stamps are added to the public list to prevent replay attacks. In addition, it uses a predetermined schedule of updating seeds for a pseudorandom generator [40].

\subsection{IoT for COVID-19 Diagnosis}

IoT technologies can improve the pace, accuracy, and efficiency of the COVID-19 diagnosis and treatment process [46]. The authors in [47] have integrated a COVID-19 Intelligent Diagnosis and Treatment Assistant Program into a cloud-based application. The application utilizes electronic medical records and machine learning techniques for automated diagnosis. It can assist the health authority by information collection from patients, managing coordination as well as enabling self-diagnosis. The application is based on fifth-generation (5G) technologies, incorporating the high bandwidth and higher communication efficiencies of such networks. It allows better coordination between diagnosis and treatment through WeChat and establishes a common platform for doctors, medical scientists, and health workers [48].

\subsection{IoT for Telemedicine Services during COVID-19}

To prevent the spread of the disease, adequate healthcare must be provided remotely to patients infected with COVID-19 [49-52]. Many health authorities are focusing on developing chatbots for automated screening of the disease and schedule follow-ups [53]. Robotic telemedicine carts (such as 
Vici InTouch) can be used for the remote monitoring of patients in quarantine without the physical presence of the health workers. A number of hospitals in the USA monitor patients in intensive care units through the use of bidirectional audio and video communications [51].

\subsection{IoT-Enabled Wearable Technologies for Predicting COVID-19}

The advancement of IoT-enabled wearables has promoted a number of mobile applications for monitoring physiological parameters such as heart rate, temperature, and sleep cycles [51]. These devices or sensors can be used and integrated with machine learning technologies to predict the different phases of COVID-19 infection. The automated monitoring of temperature and blood oxygen levels can generate alarms to prioritize attention from hospital staff. There has been a number of studies such as Digital Engagement and Tracking for Early Control and Treatment or Tempredict through the use of data from Fitbit or Apple Watch to match the heart rate and activity pattern with that reported from the patients infected with COVID-19 [51].

\subsection{Drone and UAV to Fight the Pandemic}

The contagious nature of COVID-19 will compel a majority of the health-monitoring activities to take place remotely, causing significant stress on the existing Wi-Fi connections. Thus, alternative communication technologies such as Long Range Wide Area Network (LoRaWAN) have received significant focus. For example, unmanned aerial vehicles (UAVs) have been proposed to improve the information exchange reliability between LoRA devices attached to the infected users and the base station. The approach makes use of the distributed topology control algorithm to update the UAV topology based on the movement of the LoRA end devices, which improves the packet reception rate at the base station [54].

Similarly, drone technologies and robotics can be significantly useful for resolving many different technological challenges arising from the pandemic [4]. Given that many people will have to stay in quarantine for a long time period irrespective of their infection status, there can be a number of applications where drones can be useful such as drone-based food delivery, the automated delivery of parcels [55], as well as providing assistance with the handling of clinical microbiology samples. Drones can also help with the delivery of life-saving medicines in remote hospitals [56]. Robots can be used in hospitals and other premises to remind users about social distancing and the use of masks/gloves. They can also be trained for facial emotion recognition to identify health workers in stress who need further attention [4]. Table 3 outlines the aforementioned applications of IoT in managing COVID-19 pandemic.

Table 3. Summary of the research contributions in IoT for COVID-19 applications.

\begin{tabular}{|c|c|c|c|}
\hline Paper/Project Name & Reference No. & Domain & Contribution \\
\hline $\begin{array}{l}\text { Madurai Elavarasan, R., and Pugazhendhi, } \\
\text { R. (2020). Restructured society and } \\
\text { environment: A review on potential } \\
\text { technological strategies to control the } \\
\text { COVID-19 pandemic. }\end{array}$ & [4] & $\begin{array}{l}\text { Drone and UAV to achieve } \\
\text { protection against } \\
\text { the pandemic }\end{array}$ & $\begin{array}{l}\text { Reviews the potential technologies } \\
\text { such as AI, ML and IoT in } \\
\text { healthcare sector for forecast of } \\
\text { epidemic, disease investigation, } \\
\text { medicine delivery, etc. }\end{array}$ \\
\hline $\begin{array}{l}\text { Justin Chan, Dean Foster, Shyam Gollakota, } \\
\text { Eric Horvitz†, Joseph Jaeger, Sham Kakade, } \\
\text { Tadayoshi Kohno, John Langford, Jonathan } \\
\text { Larson, Sudheesh Singanamalla, Jacob } \\
\text { Sunshine, Stefano Tessaro. "PACT: } \\
\text { Privacy-Sensitive Protocols And } \\
\text { Mechanisms for Mobile Contact Tracing". }\end{array}$ & {$[40]$} & $\begin{array}{l}\text { Existing contact } \\
\text { tracing mechanisms }\end{array}$ & $\begin{array}{l}\text { Implementation of IoT technology } \\
\text { to improve the efficiency of } \\
\text { contact tracing. }\end{array}$ \\
\hline $\begin{array}{l}\text { Hyunghoon Cho, Daphne Ippolito, Yun } \\
\text { William Yu. "Contact Tracing Mobile Apps } \\
\text { for COVID-19: Privacy Considerations and } \\
\text { Related Trade-offs". }\end{array}$ & [41] & $\begin{array}{l}\text { Contact tracing using } \\
\text { TraceTogether }\end{array}$ & $\begin{array}{l}\text { Demonstration of IoT-enabled } \\
\text { mobile app for contact tracing } \\
\text { of COVID- } 19 \text {. }\end{array}$ \\
\hline
\end{tabular}


Table 3. Cont.

\begin{tabular}{|c|c|c|c|}
\hline Paper/Project Name & Reference No. & Domain & Contribution \\
\hline $\begin{array}{l}\text { Qiang Tang. "Privacy-Preserving Contact } \\
\text { Tracing: current solutions and } \\
\text { open questions". }\end{array}$ & [42] & $\begin{array}{l}\text { Efficient privacy-preserving } \\
\text { contact tracing }\end{array}$ & $\begin{array}{l}\text { Discussion on privacy preserving } \\
\text { contact tracing solution and } \\
\text { their challenges. }\end{array}$ \\
\hline $\begin{array}{l}\text { Prof. Carmela Troncoso et al., } \\
\text { "Decentralized Privacy-Preserving } \\
\text { Proximity Tracing". }\end{array}$ & [43] & Contact tracing using DP3T & $\begin{array}{l}\text { Demonstration of a decentralized } \\
\text { privacy preserving proximity } \\
\text { tracing solution. }\end{array}$ \\
\hline $\begin{array}{l}\text { Thamer Altuwaiyan, Mohammad Hadian, } \\
\text { and Xiaohui Liang. "EPIC: Efficient } \\
\text { Privacy-preserving Contact Tracing for } \\
\text { Infection Detection". }\end{array}$ & {$[44]$} & $\begin{array}{l}\text { Efficient privacy-preserving } \\
\text { contact tracing }\end{array}$ & $\begin{array}{l}\text { Demonstration of a privacy } \\
\text { preserving contact tracing solution } \\
\text { using Bluetooth technology. }\end{array}$ \\
\hline $\begin{array}{l}\text { Christoph, Michael and Daniel Günther. } \\
\text { "Tracing Contacts to Control the } \\
\text { COVID-19 Pandemic". }\end{array}$ & [45] & $\begin{array}{l}\text { Contact categorization for } \\
\text { contact tracing }\end{array}$ & $\begin{array}{l}\text { Demonstration of contact } \\
\text { categorization based on } \\
\text { Bluetooth messages. }\end{array}$ \\
\hline $\begin{array}{l}\text { Bai, L., Yang, D., Wang, X., Tong, L., Zhu, X., } \\
\text { Bai, C., and Powell, C. A. (2020). Chinese } \\
\text { experts' consensus on the Internet of } \\
\text { Things-aided diagnosis and treatment of } \\
\text { coronavirus disease } 2019 .\end{array}$ & [47] & IoT for COVID-19 diagnosis & $\begin{array}{l}\text { Implementation of cloud-based } \\
\text { IoT enabled applications for } \\
\text { diagnostics and treatment } \\
\text { of COVID-19. }\end{array}$ \\
\hline $\begin{array}{l}\text { Christian Montag, Benjamin Becker and } \\
\text { Chunmei Gan. "The Multipurpose } \\
\text { Application WeChat: A Review on } \\
\text { Recent Research". }\end{array}$ & [48] & IoT for COVID-19 diagnosis & $\begin{array}{l}\text { Application of WeChat app for } \\
\text { better coordination between } \\
\text { diagnosis and treatment of } \\
\text { COVID-19 for doctors, medical } \\
\text { scientists, and health workers. }\end{array}$ \\
\hline $\begin{array}{l}\text { Kapoor, A., Guha, S., Kanti Das, M., } \\
\text { Goswami, K. C., and Yadav, R. (2020). } \\
\text { Digital Healthcare: The only solution for } \\
\text { better healthcare during } \\
\text { COVID-19 pandemic? }\end{array}$ & [51] & $\begin{array}{l}\text { IoT for telemedicine services } \\
\text { during COVID-19, IoT-enabled } \\
\text { wearable technologies for } \\
\text { predicting COVID-19 }\end{array}$ & $\begin{array}{l}\text { Demonstration of IoT-enabled } \\
\text { remote and wearable monitoring } \\
\text { solution for the } \\
\text { COVID-19 epidemic. }\end{array}$ \\
\hline $\begin{array}{l}\text { Saraereh, O. A., Alsaraira, A., Khan, I., and } \\
\text { Uthansakul, P. (2020). Performance } \\
\text { Evaluation of UAV-Enabled LoRa Networks } \\
\text { for Disaster Management Applications. }\end{array}$ & [54] & $\begin{array}{l}\text { Drone and UAV to fight the } \\
\text { pandemic }\end{array}$ & $\begin{array}{l}\text { Application of Long Range Wide } \\
\text { Area Network (LoRaWAN) } \\
\text { technology for remote monitoring } \\
\text { of COVID-19 epidemic activity. }\end{array}$ \\
\hline
\end{tabular}

\section{Potential Risk of Intelligent Techniques}

Although AI, ML, and IoT technologies have a huge potential to improve as well as automate the COVID tracing, management, and associated healthcare applications, the risks of their implementation cannot be overlooked. This section discusses the potential vulnerabilities and implications of the incorrect decisions made by AI, ML, and IoT technologies.

\subsection{Potential Risks of $A I$ and ML}

AI and ML are data-driven techniques where the decisions are made based on the model developed completely from data or information. There is a saying: "garbage in, garbage out". Now, any decision made based on the AI and ML will be biased if the input data is wrong or falsified. During COVID-19, information management has been highlighted as a critical challenge, as there is lots of wrong and false information propagating in the media. Therefore, policy decisions, as well as patient monitoring and diagnosis, could be biased or wrongly conducted because of the wrong input. Therefore, a significant amount of risk is associated during the automation of the AI and ML-based systems [57]. GAN, based on a class of deep learning and machine learning class, can create new images or datasets with the same statistics as the training dataset. As a result, GAN can create images that never existed before. Consequently, an adversary can use this learning model to generate fake CT scan data or other medical imaging data and images. Mirsky et al. show that a stealthy attack can fool the radiologists with a high success rate, e.g., cancer injection has a success rate of $99.2 \%$, while cancer removal has $95.8 \%$ when radiologists have no prior knowledge about the attack [57,58]. 


\subsection{Potential Risks of IoT}

The existing IoT applications certainly have some limitations in terms of privacy and security. The contact tracing methods currently used by different countries pose significant challenges in terms of security risks. For example, the mechanism used in TraceTogether requires a high level of trust to be placed upon government officials, as they can identify sensitive information of persons infected with COVID-19. Thus, the TraceTogether app cannot offer privacy preserving solutions for the infected group, although in general, it can keep information secured from third parties [38]. The decentralized solution in DP3T can bring new types of privacy attacks in the scenario, including backend impersonation, false report, replay attack, and relay attack [59]. Data breaches in healthcare systems can massively impact the treatment process and patient well-being, which have emerged as a serious problem in modern healthcare systems with increasing hacking attacks [60]. IoT-based applications can enhance this vulnerability a lot more due to their dependence on data extracted from connected networks. Not every age group in the society will have the same level of technology readiness, which poses additional challenges in the adoption of IoT-based applications in healthcare, as identified in a survey from older/senior citizens [61].

\subsection{Enhancing the Privacy and Security of AI and IoT Techniques}

Since privacy and security are the key challenges of AI and IoT techniques, given the sensitivity of the revealed information and associated implications, it is highly important to ensure appropriate measures for preserving privacy and security in the AI and IoT applications. In general, these measures need to achieve privacy from the "trusted" authority, attackers as well as other users of the app. For COVID-19 contact tracing applications, polling can be a mechanism to protect the privacy of the users infected with COVID-19 from the non-infected users, where non-infected users can poll the health authority regularly to check if they have been in close contact with an infected user. Privacy from the health authority can be achieved using private messaging systems [62] and private set intersection protocols [63]. However, these protocols can be computationally expensive, so a trade-off needs to be designed between security and computational efficiency [41]. The integrity of the COVID-positive reports can be enhanced by including verification keys that combine a memo to the report through cryptography. The memo can list a summary of users' symptoms as well as certificates from third-party health services [40].

The incorporation of innovative technologies to secure information sharing during a pandemic such as COVID-19 can be highly effective. In this regard, appropriate standardization methods for new communication and network protocols are required for their seamless integration in healthcare systems [64]. Since IoT applications enable widespread access to system monitoring data, new data-driven security techniques can be developed to build predictive ML models to detect IoT attacks [65]. For secured information sharing among multiple entities in the healthcare sector, distributed ledger technologies and blockchain can ensure the information integrity without involving a third party, which allows privacy-preserving solutions for smart healthcare systems [66].

\section{Conclusions}

In this paper, a range of medical sector applications such as drug delivery and infection detection based on AI and ML techniques have been elaborated. Moreover, AI and ML applications for forecasting disease trends and developing social awareness programs have been outlined. In addition, the recent advancements in IoT for tracing and reporting patients infected with COVID-19, as well as the potential applications in automated monitoring through wearables technologies have been reviewed. The applications of blockchain technologies for decentralized decision making in healthcare systems during COVID-19 scenarios have been outlined. Security and privacy aspects of the AI, ML, and IoT technologies have also been elaborated, along with their impact on the critical policy-making 
operations. Future studies will be performed to extend the findings for developing new algorithms for IoT-aided COVID-19 health-monitoring applications.

Author Contributions: Conceptualization, A.A. and A.M.T.O.; methodology, S.N.I.; software, A.N.M.; validation, S.M.A.A.A., and S.N.I.; formal analysis, S.M.A.A.A.; investigation, A.A. and A.N.M.; resources, A.M.T.O.; data curation, S.M.A.A.A; writing — original draft preparation, S.M.A.A.A. and S.N.I.; writing—review and editing, A.A.; visualization, A.A.A. and S.N.I.; supervision, A.N.M.; project administration, A.M.T.O.; All authors have read and agreed to the published version of the manuscript.

Funding: This research received no external funding.

Conflicts of Interest: The authors declare no conflict of interest.

\section{References}

1. Timeline: WHO's COVID-19 Response. Available online: https://www.who.int/emergencies/diseases/novelcoronavirus-2019/interactive-timeline?gclid=CjwKCAiA2O39BRBjEiwApB2Ikh-fQFKjiYNKczrHGfWVpPVYkuNEh6d53pNhA8fEM1WYjtAfpaSERoC0lkQAvD_BwE\#event-115 (accessed on 24 August 2020).

2. WHO Coronavirus Disease (COVID-19) Dashboard. Available online: https://covid19.who.int/ (accessed on 24 August 2020).

3. COVID-19 Coronavirus Pandemic, Worldometer. Available online: https://www.worldometers.info/ coronavirus/ (accessed on 24 August 2020).

4. Elavarasan, R.M.; Pugazhendhi, R. Restructured society and environment: A review on potential technological strategies to control the COVID-19 pandemic. Sci. Total Environ. 2020, 725, 138858. [CrossRef]

5. Bullock, J.; Luccioni, A.; Hoffmann Pham, K.; Lam, C.S.N.; Luengo-Oroz, M. Mapping the landscape of Artificial Intelligence applications against COVID-19. arXiv 2020, arXiv:2003.11336.

6. COVID-19 Open Research Dataset (CORD-19). Semantic Scholar. Published 2020. Available online: https://pages.semanticscholar.org/coronavirus-research (accessed on 30 March 2020).

7. Alwashmi, M.F. The Use of Digital Health in the Detection and Management of COVID-19. Int. J. Environ. Res. Public Health 2020, 17, 2906. [CrossRef]

8. One Healthcare Cloud: Many Imaging Solutions, Cimar. Available online: www.cimar.co.uk (accessed on 25 November 2020).

9. Hare, S.; Rodrigues, J.; Jacob, J.; Edey, A.; Devaraj, A.; Johnstone, A.; McStay, R.; Nair, A.; Robinson, G. A UK-wide British Society of Thoracic Imaging COVID-19 imaging repository and database: Design, rationale and implications for education and research. Clin. Radiol. 2020, 75, 326-328. [CrossRef]

10. Shobha, G.; Rangaswamy, S. Chapter 8-Machine Learning. In Handbook of Statistics; Elsevier: Amsterdam, The Netherlands, 2018; Volume 38, pp. 197-228.

11. Bogoch, I.I.; Watts, A.; Thomas-Bachli, A.; Huber, C.; Kraemer, M.U.G.; Khan, K. Pneumonia of unknown aetiology in Wuhan, China: Potential for international spread via commercial air travel. J. Travel Med. 2020, 27, 1-3. [CrossRef]

12. Shi, F.; Wang, J.; Shi, J.; Wu, Z.; Wang, Q.; Tang, Z.; He, K.; Shi, Y.; Shen, D. Review of Artificial Intelligence Techniques in Imaging Data Acquisition, Segmentation and Diagnosis for COVID-19. IEEE Rev. Biomed. Eng. 2020, 1. [CrossRef]

13. Meng, L. Review: Chest CT Features and Their Role in COVID-19. Radiology of Infectious Diseases. Available online: www.sciencedirect.com (accessed on 30 March 2020).

14. Loey, M.; Smarandache, F.M.; Khalifa, N.E. Within the Lack of Chest COVID-19 X-ray Dataset: A Novel Detection Model Based on GAN and Deep Transfer Learning. Symmetry 2020, 12, 651. [CrossRef]

15. Öztürk, Ş.; Özkaya, U.; Barstuğan, M. Classification of Coronavirus (COVID-19) from X-ray and CT images using shrunken features. Int. J. Imaging Syst. Technol. 2020. [CrossRef] [PubMed]

16. Kassania, S.H.; Kassasnib, P.H.; Wesolowskic, M.J.; Schneider, K.A.; Deters, R. Automatic Detection of Coronavirus Disease (COVID-19) in X-ray and CT Images: A Machine Learning Based Approach. arXiv 2020, arXiv:2004.10641.

17. Beck, B.R.; Shin, B.; Choi, Y.; Park, S.; Kang, K. Predicting commercially available antiviral drugs that may act on the novel coronavirus (SARS-CoV-2) through a drug-target interaction deep learning model. Comput. Struct. Biotechnol. J. 2020, 18, 784-790. [CrossRef] [PubMed] 
18. Magar, R.; Yadav, P.; Farimani, A.B. Potential Neutralizing Antibodies Discovered for Novel Corona Virus Using Machine Learning. arXiv 2020, arXiv:2003.08447.

19. Zhong, L.; Mu, L.; Li, J.; Wang, J.; Yin, Z.; Liu, D. Early Prediction of the 2019 Novel Coronavirus Outbreak in the Mainland China Based on Simple Mathematical Model. IEEE Access 2020, 8, 51761-51769. [CrossRef] [PubMed]

20. Vaishya, R.; Javaid, M.; Khan, I.H.; Haleem, A. Artificial Intelligence (AI) applications for COVID-19 pandemic. Diabetes Metab. Syndr. Clin. Res. Rev. 2020, 14, 337-339. [CrossRef] [PubMed]

21. Magdon-Ismail, M. Machine Learning the Phenomenology of COVID-19 From Early Infection Dynamics. arXiv 2020, arXiv:2003.07602.

22. Ghoshal, B.; Tucker, A. Estimating Uncertainty and Interpretability in Deep Learning for Coronavirus (COVID-19) Detection. arXiv 2020, arXiv:2003.10769v3.

23. Ndiaye, B.M.; Tendeng, L.; Seck, D. Analysis of the COVID-19 pandemic by SIR model and machine learning technics for forecasting. arXiv 2020, arXiv:2004.01574v1.

24. Batra, R.; Chan, H.; Kamath, G.; Ramprasad, R.; Cherukara, M.J.; Sankaranarayanan, S. Screening of Therapeutic Agents for COVID-19 using Machine Learning and Ensemble Docking Simulations. arXiv 2020, arXiv:2004.03766v1.

25. Magri, L.; Khoa Doan, N.A. First-principles machine learning modelling of COVID-19. arXiv 2020, arXiv:2004.09478v1.

26. McCall, B. COVID-19 and artificial intelligence: Protecting health-care workers and curbing the spread. Lancet Digit. Health 2020, 2, e166-e167. [CrossRef]

27. Mashamba-Thompson, T.P.; Crayton, E.D. Blockchain and Artificial Intelligence Technology for Novel Coronavirus Disease 2019 Self-Testing. Diagnostics 2020, 10, 198. [CrossRef]

28. Liu, D.; Clemente, L.; Poirier, C.; Ding, X.; Chinazzi, M.; Davis, J.T.; Vespignani, A.; Santillana, M. A machine learning methodology for real-time forecasting of the 2019-2020 COVID-19 outbreak using Internet searches, news alerts, and estimates from mechanistic models. arXiv 2020, arXiv:2004.04019v1.

29. Qi, X.; Mei, G.; Cuomo, S.; Xiao, L. A network-based method with privacy-preserving for identifying influential providers in large healthcare service systems. Futur. Gener. Comput. Syst. 2020, 109, 293-305. [CrossRef] [PubMed]

30. Soures, N.; Chambers, D.; Carmichael, Z.; Daram, A.; Shah, D.P.; Clark, K.; Potter, L.; Kudithipudi, D. SIRNET: Understanding Social Distancing Measures with Hybrid Neural Network Model for COVID-19 Infectious Spread. arXiv 2020, arXiv:2004.10376v1.

31. Li, L.; Zhang, Q.; Wang, X.; Zhang, J.J.; Wang, T.; Gao, T.-L.; Duan, W.; Tsoi, K.K.-F.; Wang, F.-Y. Characterizing the Propagation of Situational Information in Social Media During COVID-19 Epidemic: A Case Study on Weibo. IEEE Trans. Comput. Soc. Syst. 2020, 7, 556-562. [CrossRef]

32. Kuziemski, M.; Misuraca, G. AI governance in the public sector: Three tales from the frontiers of automated decision-making in democratic settings. Telecommun. Policy 2020, 44, 101976. [CrossRef]

33. Pandey, R.; Gautam, V.; Jain, C.; Syal, P.; Sharma, H.; Bhagat, K.; Pal, R.; Dhingra, L.S.; Arushi; Patel, L.; et al. A Machine Learning Application for Raising WASH Awareness in the Times of COVID-19 Pandemic. arXiv 2020, arXiv:2003.07074.

34. Rashid, M.T.; Wang, D. CovidSens: A Vision on Reliable Social Sensing based Risk Alerting Systems for COVID-19 Spread. arXiv 2020, arXiv:2004.04565v1.

35. Bell, J.; Butler, D.; Hicks, C.; Crowcroft, J. TraceSecure: Towards Privacy Preserving Contact Tracing. arXiv 2020, arXiv:2004.04059v1.

36. Roomp, K.; Oliver, N. ACDC-Tracing: Towards Anonymous Citizen-Driven Contact Tracing. arXiv 2020, arXiv:2004.07463.

37. Lorch, L.; Trouleau, W.; Tsirtsis, S.; Szanto, A.; Schölkopf, B.; Gomez-Rodriguez, M. A Spatiotemporal Epidemic Model to Quantify the Effects of Contact Tracing, Testing, and Containment. arXiv 2020, arXiv:2004.07641v1.

38. Venkateswaran, J.; Damani, O. Effectiveness of Testing, Tracing, Social Distancing and Hygiene in Tackling Covid-19 in India: A System Dynamics Model. arXiv 2020, arXiv:2004.08859.

39. Kim, H.; Paul, A. Contact Tracing: A game of big numbers in the time of COVID-19. arXiv 2020, arXiv:2004.10762v1. [CrossRef] 
40. Chan, J.; Foster, D.; Gollakota, S.; Horvitz, E.; Jaeger, J.; Kakade, S.; Kohno, T.; Langford, J.; Larson, J.; Singanamalla, S.; et al. PACT:Privacy-Sensitive Protocols And Mechanisms for Mobile Contact Tracing. arXiv 2020, arXiv:2004.03544v3.

41. Cho, H.; Ippolito, D.; Yu, Y.W. Contact Tracing Mobile Apps for COVID-19:Privacy Considerations and Related Trade-offs. arXiv 2020, arXiv:2003.11511v2.

42. Tang, Q. Privacy-Preserving Contact Tracing: Current solutions and open questions. arXiv 2020, arXiv:2004.06818v2.

43. Troncoso, C.; Payer, M.; Hubaux, J.-P.; Salathé, M.; Larus, J.; Bugnion, E.; Lueks, W.; Stadler, T.; Pyrgelis, A.; Antonioli, D.; et al. Decentralized Privacy-Preserving Proximity Tracing. arXiv 2020, arXiv:2005.12273.

44. Altuwaiyan, T.; Hadian, M.; Liang, X. EPIC: Efficient Privacy-Preserving Contact Tracing for Infection Detection. In Proceedings of the 2018 IEEE International Conference on Communications (ICC), Kansas City, MO, USA, 24-28 May 2018.

45. Michael, C.; Günther, D. Tracing Contactsto Control the COVID-19 Pandemic. arXiv 2020, arXiv:2004.00517v1.

46. Rahman, S.; Peeri, N.C.; Shrestha, N.; Zaki, R.; Haque, U.; Ab Hamid, S.H. Defending against the Novel Coronavirus (COVID-19) outbreak: How can the Internet of Things (IoT) help to save the world? Health Policy Technol. 2020, 9, 136-138. [CrossRef]

47. Bai, L.; Yang, D.; Wang, X.; Tong, L.; Zhu, X.; Zhong, N.; Bai, C.; Powell, C.A.; Chen, R.; Zhou, J.; et al. Chinese experts' consensus on the Internet of Things-aided diagnosis and treatment of coronavirus disease 2019 (COVID-19). Clin. eHealth 2020, 3, 7-15. [CrossRef]

48. Montag, C.; Becker, B.; Gan, C. The Multipurpose Application WeChat: A Review on Recent Research. Front. Psychol. 2018, 9, 2247. [CrossRef]

49. Yang, T.; Gentile, M.; Shen, C.-F.; Cheng, C. Combining Point-of-Care Diagnostics and Internet of Medical Things (IoMT) to Combat the COVID-19 Pandemic. Diagnostics 2020, 10, 224. [CrossRef] [PubMed]

50. Torous, J.; Keshavan, M. COVID-19, mobile health and serious mental illness. Schizophr. Res. 2020, 218 , 36-37. [CrossRef] [PubMed]

51. Kapoor, A.; Guha, S.; Das, M.; Goswami, K.C.; Yadav, R. Digital healthcare: The only solution for better healthcare during COVID-19 pandemic? Indian Hear. J. 2020, 72, 61-64. [CrossRef] [PubMed]

52. Ying, W.; Qian, Y.; Kun, Z. Drugs supply and pharmaceutical care management practices at a designated hospital during the COVID-19 epidemic. Res. Soc. Adm. Pharm. 2020. [CrossRef] [PubMed]

53. Gupta, M.; Abdelsalam, M.; Mittal, S. Enabling and Enforcing Social Distancing Measures using Smart City and ITS Infrastructures:A COVID-19 Use Case. arXiv 2020, arXiv:2004.09246v1.

54. Saraereh, O.A.; Alsaraira, A.; Khan, I.; Uthansakul, P. Performance Evaluation of UAV-Enabled LoRa Networks for Disaster Management Applications. Sensors 2020, 20, 2396. [CrossRef] [PubMed]

55. Kellermann, R.; Biehle, T.; Fischer, L. Drones for parcel and passenger transportation: A literature review. Transp. Res. Interdiscip. Perspect. 2020, 4. [CrossRef]

56. Mesar, T.; Lessig, A.; King, D.R. Use of Drone Technology for Delivery of Medical Supplies During Prolonged Field Care. J. Spéc. Oper. Med. 2018, 18, 34-35.

57. Chu, L.C.; Anandkumar, A.; Shin, H.C.; Fishman, E.K. The Potential Dangers of Artificial Intelligence for Radiology and Radiologists. J. Am. Coll. Radiol. 2020, 17, 1309-1311. [CrossRef]

58. Mirsky, Y.; Mahler, T.; Shelef, I.; Elovici, Y. CT-GAN: Malicious Tampering of 3D Medical Imagery using Deep Learning. In Proceedings of the 28th USENIX Security Symposium, Santa Clara, CA, USA, 14-16 August 2019; pp. 461-478.

59. Vaudenay, S. Analysis of DP3T Between Scylla and Charybdis. Available online: https://infoscience.epfl.ch/ record/277808/files/dp3t-ana.pdf (accessed on 10 July 2020).

60. Seh, A.H.; Zarour, M.; Alenezi, M.; Sarkar, A.K.; Alenezi, M.; Kumar, R.; Agrawal, A. Healthcare Data Breaches: Insights and Implications. Healthcare 2020, 8, 133. [CrossRef]

61. Wang, S.; Bolling, K.; Mao, W.; Reichstadt, J.; Jeste, D.; Kim, H.-C.; Nebeker, C. Technology to Support Aging in Place: Older Adults' Perspectives. Healthcare 2019, 7, 60. [CrossRef] [PubMed]

62. Van den Hooff, J.; Lazar, D.; Zaharia, M.; Zeldovich, N. Vuvuzela: Scalable private messaging resistant to traffic analysis. In Proceedings of the 25th Symposium on Operating Systems Principles (SOSP '15); Association for Computing Machinery: New York, NY, USA, 2015; pp. 137-152.

63. Freedman, M.J.; Nissim, K.; Pinkas, B. Efficient Private Matching and Set Intersection. Lecture Notes Comput. Sci. 2004, 1-19. [CrossRef] 
64. Allam, Z.; Jones, D.S. On the Coronavirus (COVID-19) Outbreak and the Smart City Network: Universal Data Sharing Standards Coupled with Artificial Intelligence (AI) to Benefit Urban Health Monitoring and Management. Healthcare 2020, 8, 46. [CrossRef] [PubMed]

65. Wheelus, C.; Zhu, X. IoT Network Security: Threats, Risks, and a Data-Driven Defense Framework. IoT 2020, 1, 259-285. [CrossRef]

66. Liu, H.; Crespo, R.G.; Martínez, O.S. Enhancing Privacy and Data Security across Healthcare Applications Using Blockchain and Distributed Ledger Concepts. Healthcare 2020, 8, 243. [CrossRef]

Publisher's Note: MDPI stays neutral with regard to jurisdictional claims in published maps and institutional affiliations.

(C) 2020 by the authors. Licensee MDPI, Basel, Switzerland. This article is an open access article distributed under the terms and conditions of the Creative Commons Attribution (CC BY) license (http://creativecommons.org/licenses/by/4.0/). 